\title{
Il patrimonio fotografico Alinari: excursus storico e questioni attuali
}

\begin{abstract}
Following the purchase by the Regione Toscana (2019), various possibilities arise for the reorganization and cultural destination of the Alinari photographic heritage. The essay outlines a historical excursus intended to illustrate the methods, phases and type of materials with which such an important archival-museum complex has been formed over time, always playing, in different ways, a particularly important role in Italian and international visual culture.
\end{abstract}

\section{Keywords}

PHOTOGRAPHIC HERITAGE; ALINARI COMPANY; MUSEUM;

PHOTOGRAPHIC ARCHIVES; PUBLIC HERITAGE; REGION TUSCANY;

FLORENCE; VISUAL CULTURE

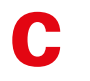

on la Legge regionale 13 novembre 2019, n. 65, capo IV, art. 55 , l'intero patrimonio fotografico della Società Fratelli Alinari IDEA è stato acquistato dalla Regione Toscana, "al fine di assicurare il mantenimento e l'integrità" del patrimonio stesso, nonché "le migliori condizioni di utilizzazione e fruizione pubblica anche mediante l'attività di riproduzione e circolazione delle immagini digitali" -1.

Questo atto è giunto al termine di un prolungato periodo di crisi economica che aveva coinvolto Alinari, come altre aziende del settore, negli ultimi anni, e che era culminato fra la fine del 2018 e l'inizio del 2019 con la decisione della proprietà di vendere prima (a privati) l'edificio storico sede della ditta fin dal 1863, e poi anche (alla Regione Toscana) il patrimonio fotografico.

Si tratta, come si può capire, di un evento di importanza straordinaria per quanto riguarda il patrimonio fotografico italiano.

Il complesso archivistico-documentario-museale ricompreso sotto il nome Alinari in realtà va molto oltre il lascito dei fondatori della Ditta, e costituisce uno degli archivi fotografici più importanti al mondo, per qualità e quantità dei reperti conservati. 
Per quanto la Regione Toscana non abbia acquisito l'azienda in quanto tale, ma solo il patrimonio storico, occorre tener presente che fino a pochi mesi fa e per i quasi 170 anni precedenti gli Alinari erano stati una ditta privata che operava sul mercato, un mercato peraltro influenzato non solo da variabili economiche, ma anche tecnologiche e culturali; e che adattandosi appunto a tali variabili aveva più volte cambiato, anche profondamente, modelli operativi e tipologie produttive. Tutto ciò si riflette nel patrimonio attualmente trasmesso alla Regione Toscana, che è molto diversificato e per certi aspetti disomogeneo, quanto alle tipologie di materiali documentari conservati. A ciò si aggiunge il fatto che tutto il materiale è attualmente inscatolato presso un deposito provvisorio, dovrà essere ricollocato in una nuova sede, $\mathrm{e}$ dovrà quindi essere oggetto di una delicata e complessa operazione di risistemazione, dove l'articolazione interna del precedente archivio potrà essere rideterminata.

Un ulteriore aspetto importante relativo ai problemi che sorgeranno in seguito all'acquisizione pubblica (peraltro benemerita) del patrimonio, è che la ditta ha operato quasi sempre non solo sul piano economico, ma anche sul piano culturale, realizzando oltre alle produzioni vere e proprie in campo fotografico, anche una serie di attività (espositive, editoriali, museali) e intrattenendo una serie di rapporti, di interazioni che hanno esercitato una notevole influenza sui caratteri e la diffusione della cultura fotografica in Italia. Il problema che si pone quindi non è solo quello di gestire un archivio con procedure standard dal punto di vista della conservazione e tutela del materiale e della fruizione da parte del pubblico, ma è quello più complesso di elaborare strutture e modelli operativi per innestare sui diversi fondi archivistici, sulle raccolte, sulla documentazione e i musealia esistenti un'attività di riorganizzazione, di valorizzazione e di promozione culturale adeguata al significato e all'importanza di questo patrimonio documentario.

Questo articolo vuole essere un contributo a impostare sul piano storiografico tali problemi, cercando di ricostruire le caratteristiche peculiari, il significato che questo patrimonio ha avuto in passato e che può avere attualmente, attraverso un breve excursus storico sulle varie fasi che hanno portato alla situazione attuale ${ }^{-2}$.

\section{Le origini (1852-1870)}

La storia degli Alinari inizia convenzionalmente nel 1852 a Firenze. La particolarità che contraddistingue l'impresa Alinari fin dalle origini, e che sarà poi un tratto costitutivo di tutta la sua vita successiva, è il fatto che il suo fondatore, il giovane Leopoldo Alinari, arriva alla fotografia in virtù della sua posizione iniziale di apprendista all'interno della calcografia di Luigi Bardi, titolare di una delle più note e affermate botteghe di incisione su rame nella Firenze dell'Ottocento. Nel seguito della sua attività Alinari porterà sempre l'impronta di questo periodo di formazione, che lo pone su una linea di continuità con una tradizione figurativa alta e consolidata, e soprattutto con il tema della rappresentazione 
del patrimonio artistico, monumentale e paesaggistico italiano per un pubblico internazionale.

La scelta di Bardi di incaricarlo di sviluppare la nuova tecnica, permettendogli di formarsi anche con viaggi all'estero, offre al giovane lavorante opportunità straordinarie, che lo portano nel giro di pochi anni a mettersi in proprio fino, nel 1863, a inaugurare per la ditta una sede straordinariamente ampia e funzionale, sostanzialmente la stessa giunta fino ai giorni nostri, costata una cifra ragguagliabile a diversi milioni di euro attuali, a dimostrazione di come l'attività dell'impresa fosse già economicamente fiorente e in pieno sviluppo.

Casi di fiorenti guadagni da parte dei fotografi in quell'epoca non erano infrequenti, specie in relazione ad alcune innovazioni tecniche che sviluppavano nuove "mode" a larga diffusione, come la stereoscopia o la carte de visite.

Ma perché proprio gli Alinari? È vero che pur avendo anch'essi percorso quelle strade molto redditizie, si erano fin dall'inizio specializzati in un settore ben preciso, che era quello della riproduzione dei monumenti, delle architetture e delle opere d'arte; ma in quel periodo in Italia erano attivi, nelle "cento città", tutte ricche di capolavori d'arte, che costellavano il Bel Paese, molti fotografi che svolgevano lo stesso tipo di lavoro e talvolta non avevano molto da invidiare agli Alinari sul piano tecnico, della qualità, del pregio delle riprese e anche del gradimento del pubblico e della critica. Perché non loro, al posto o accanto ai fratelli fiorentini?

\section{La dimensione internazionale del patrimonio Alinari (1870-1890)}

La risposta, essenziale per capire questo successo e quindi il significato e il valore della produzione conservata nel loro archivio, stava in una particolare strategia imprenditoriale-culturale, e la fornirono, già al tempo, gli Alinari stessi. Nel 1872, di fronte alla Commissione dell'Inchiesta Industriale, affermarono con decisione, con un certo orgoglio e anche con una certa spavalderia, che loro erano certi di guadagnare molto di più, e precisamente vari ordini di grandezza più degli altri fotografi professionisti delle altre città, grazie a un tipo di esportazione particolare. Si trattava di una tipologia di vendita che non si rivolgeva direttamente all'utente finale, ma ad agenti, a "corrispondenti" este$\mathrm{ri}^{-3}$, i quali ricevevano in conto deposito alcuni stock di fotografie per distribuirle, in forme molto flessibili e capillari, verso varie clientele, ma soprattutto verso un mercato ben preciso, quello della didattica e degli studi di storia dell'arte. Come specificarono Giuseppe e Romualdo (Leopoldo era morto nel 1865) nelle Deposizioni scritte dell'Inchiesta,

Si fa molta esportazione di fotografie italiane per l'estero, ed anzi si può asserire, senza tema di errare, che tutto quanto si fabbrica, meno impercettibile quantità, si smercia all'estero, non essendo ancora compresa l'Italia dell'importanza massima della fotografia, non solo per le scuole, ma anche per gli studi comparativi ${ }^{-4}$. 
In questa precoce vocazione verso la documentazione del patrimonio artistico italiano per il mercato internazionale sta l'originalità e la specificità dell'intervento dei Fratelli Alinari e la spiegazione del loro successo. Altri fotografi come Anderson fecero, almeno inizialmente, scelte diverse e opposte ${ }^{-\mathbf{5}}$.

La teorizzazione di questo tipo di attività fu compiuta più tardi dall'altro grande fotografo fiorentino, Carlo Brogi. Di fronte ai fotografi italiani riuniti nei primi congressi fotografici svolti a cura della Società Fotografica Italiana, Brogi affermò con molta chiarezza che oltre la distinzione tradizionale e già evidente tra fotografi dilettanti e professionisti, andava introdotta la categoria dei "fotografi editori", fra cui si poneva lui stesso, oltre ad Alinari e Anderson. La dizione "fotografi editori" non si riferiva però al fatto che tali fotografi si interessassero di editoria, né che pubblicassero fotografie, ma al fatto, molto più rilevante ai nostri fini, che questi fotografi ritenevano di editare non le fotografie, ma le opere d'arte: secondo la loro intenzione cioè, attraverso il dispositivo fotografico essi pubblicavano, ovvero rendevano accessibile ad un pubblico disseminato in tutto il mondo, il patrimonio artistico e monumentale italiano ${ }^{-6}$. In altre parole i ricchi e ampi cataloghi dei fotografi editori, che (non potendosi all'epoca pubblicare fotografie a stampa) non contenevano fotografie, ma lunghe e a volte dettagliatissime descrizioni delle opere d'arte, disseminate su tutto il territorio nazionale, indicizzate anche per autori e tipologie, permettevano a studiosi e persone colte di tutto il mondo di ottenere riproduzioni, e quindi vedere per la prima volta opere che altrimenti solo molto difficilmente avrebbero potuto conoscere o vedere direttamente o per altra via.

\section{La dimensione nazionale del patrimonio Alinari (1890-1914)}

La vocazione internazionale degli Alinari si mantenne anche per i decenni successivi, almeno fino alla Prima guerra mondiale, e in realtà, in forme un po' diverse anche in seguito. Tuttavia a partire dagli anni Novanta dell'Ottocento si sviluppò fortemente anche un'attenzione degli Alinari e degli altri grandi fotografi editori, in particolare di Carlo Brogi, verso una dimensione nazionale della loro attività.

Nel caso degli Alinari, dopo la morte di Romualdo e Giuseppe, entrambi scomparsi nel 1890, la direzione della ditta passò a Vittorio, figlio del fondatore Leopoldo.

Vittorio Alinari dette espressione esplicita e molto forte alla dimensione nazionale dell'attività della sua impresa. Riordinò infatti, e in parte rinnovò, l'archivio delle lastre adattandole ai nuovi ritrovati tecnici, specie nel campo dell'ortocromatismo; e soprattutto promosse una serie di campagne di documentazione fotografica su base sistematica a livello nazionale, inviando i propri operatori fotografi, accompagnati da un organico e complesso team organizzativo e tecnico, a coprire in maniera programmata e progressiva le varie realtà regionali italiane.

Non si trattava solo di una scelta tecnico-organizzativa o semplicemente economica: si inseriva in un contesto di promozione della cultura 
fotografica a livello nazionale, in cui erano attivi gli altri grandi fotografi editori. Ad esempio Carlo Brogi, figlio di Giacomo, con la collaborazione di Vittorio ebbe nel 1889 un ruolo fondamentale nella costituzione della Società Fotografica Italiana (SFI) ${ }^{-7}$.

Vittorio Alinari promosse iniziative editoriali di notevole importanza, come quella della "Miscellanea d'arte", poi divenuta la "Rivista d'arte" e alla cui direzione si succedettero personaggi come Igino Benvenuto Supino o Giovanni Poggi, che svolsero un importante ruolo anche come collaboratori scientifici per le attività della Ditta.

Una particolare attenzione rivolse alla pubblicazione di una edizione illustrata della Divina Commedia nel 1911, episodio ben noto che testimonia l'importanza di questo tipo di intervento ai fini della integrazione di Vittorio e della sua ditta in quella ideologia "nazionale" che costituiva il cardine della cultura del tempo ${ }^{-8}$.

Dedicò anche una attenzione notevole al paesaggio, anche con opere personali come il volume Paesaggi italici nella Divina Commedia, del 1921; fece sì che i suoi operatori non si limitassero a riprodurre, specie nel Mezzogiorno d'Italia, solo i monumenti, i paesaggi e le opere d'arte, come avevano fatto suo padre e i suoi zii, ma realizzassero anche un certo numero di fotografie di carattere etnografico/sociale, con immagini di popolani, di lavoratori poveri e degli antichi mestieri urbani delle città e dei paesi, soprattutto del sud, di "abitazioni di povera gente" - ${ }^{9}$. Considerando che anche Brogi aveva nello stesso periodo inserito in catalogo una serie di "Scene e costumi dal vero", si può concludere, come del resto aveva notato Quintavalle, che queste immagini non possono "interpretarsi come tipiche, caratteristiche, semplicemente folkloriche", ma costituiscono probabilmente un "momento iniziale di una lettura realistica delle classi subalterne" ${ }^{-10}$, ricca anche di spunti sul piano della rappresentazione antropologica del popolo italiano ${ }^{-11}$.

In questo modo Alinari e con lui gli altri fotografi editori si erano assicurati un posto di rilievo nel mondo della cultura e anche della politica italiana, e la cosa non fu affatto senza conseguenze sulle attività della Ditta. Quando, all'inizio del XX secolo, gli editori della carta stampata promossero una forte campagna contro la situazione di quasi monopolio creata dal "trust" sottoscritto da Alinari, Brogi e Anderson, e nel contempo i musei e gli enti pubblici detentori del patrimonio ottennero una legislazione che stabiliva tassazioni e oneri nei confronti dei fotografi, il ruolo dei contatti e dell'operazione di sensibilizzazione culturale condotta negli anni precedenti dette i suoi frutti, a cominciare dell'intervento del Marchese Torrigiani, vice-presidente della Camera, ma anche presidente della Società Fotografica Italiana, e di altri autorevoli esponenti della cultura e dell'alta società del tempo, che permisero di rendere in pratica inoperanti i provvedimenti di legge pur emanati. Una significativa traccia di questa congiuntura persiste negli archivi sia di Anderson che di Alinari, che di fronte alla crisi di rapporti con i musei e gli enti pubblici italiani, polemicamente dichiararono di voler allora rivolgersi a documentare le opere d'arte degli altri paesi europei. 
I cataloghi di Dresda, Parigi e Grecia, prodotti da Alinari fra il 1906 e il 1908, testimoniano di campagne fotografiche, come quella di Anderson sulla Spagna, attraverso le quali i nostri fotografi addirittura presumevano, evidentemente, di poter spostare gli interessi del loro pubblico sul patrimonio artistico di altri paesi, in funzione di pressione sul governo italiano a favore delle loro strategie imprenditoriali ${ }^{-12}$.

\section{Mercato, cultura e classe dirigente: la gestione del patrimonio Alinari fra aristocrazia e impresa (1920-1958)}

La grande guerra costituì un momento di rottura molto forte per tutta la fotografia italiana e in particolare per la ditta Alinari. Gli Alinari videro in pratica azzerarsi il volume delle loro vendite all'estero e questo indusse Vittorio Alinari a sospendere l'attività della ditta a partire già dal febbraio $1915^{-13}$.

Nel difficile clima dell'immediato dopoguerra, percorso da gravi problemi economici e sociali e da forti conflitti politici, Vittorio Alinari decise di alienare l'azienda, probabilmente per l'influenza congiunta della crisi economica, di motivi familiari, fra cui la morte del figlio Carlo, e per l'influsso del cambiamento profondo e del tramonto del clima culturale in cui aveva operato per uno sviluppo della cultura fotografica in Italia.

Dopo la fine della prima fase della storia degli Alinari come impresa familiare, l'impresa non venne rilevata da un altro imprenditore del settore, ma da una società per azioni formata da un gruppo di alcune decine di aristocratici e di notabili, soprattutto toscani, al cui vertice era il barone Luigi Ricasoli Firidolfi $-{ }^{14}$, con l'intento di non disperdere un patrimonio culturale il cui valore non poteva essere stabilito in termini soltanto economici.

Un primo "salvataggio" quindi, quello del 1920, anche se ad opera di privati, ma già in funzione di salvaguardia del patrimonio.

Fin dal primo periodo della conduzione Ricasoli (che in progresso di tempo acquisì la maggioranza delle azioni) un contributo notevole anche sul piano degli indirizzi culturali venne da intellettuali e studiosi, come Ugo Ojetti, Giovanni Poggi (che dal 1924 compare come consulente artistico stabile) e successivamente Tammaro De Marinis ${ }^{-15}$ : ma i consigli pur autorevoli di studiosi e intellettuali non potevano sostituire l'iniziativa imprenditoriale di un personaggio come Vittorio, il quale con la sua forte personalità e con l'importante rete di contatti professionali ma anche culturali e istituzionali, aveva accentrato su di sé tutta la vita dell'azienda nei suoi vari aspetti.

Nel complesso, l'impostazione dell'attività produttiva dell'azienda non cambiò in profondità. Venne promossa un'ampia attività di documentazione delle mostre d'arte, il che permetteva di agire su opere già selezionate e più facilmente accessibili; ma perdendo in sistematicità e per certi versi in originalità. Continuarono in parte anche le campagne su base geografica, e vennero ancora sviluppati i cataloghi locali e regionali, ma si manifestò già dopo pochi anni uno dei tratti caratteristici di 
questo tipo di conduzione, cioè la difficoltà, con un assetto proprietario di questo genere, a tenere il passo dell'aggiornamento tecnico proprio nel cuore dell'azienda, cioè nel reparto fotografico. Già nel 1929 il Presidente sottolineò la necessità di intervenire per migliorare il funzionamento di vari reparti, "e specialmente del reparto fotografico, che [...] è arretrato in rapporto all'attuale progresso dell'arte e al più utile andamento industriale del reparto stesso" ${ }^{16}$.

Inoltre, gli Alinari subivano la concorrenza sia dei naturali e tradizionali competitori, sia di quella della mano pubblica, che si realizzava "per l'invadente azione del Luce e dell'Istituto Poligrafico dello Stato" -17 .

Nel 1929 la crisi finanziaria che colpì il mercato statunitense, uno dei maggiori per gli Alinari, e negli anni successivi tutte le economie mondiali, provocò anche per la ditta fiorentina una prima grave crisi $^{-18}$, che trovò soluzione, nel 1933, grazie all'aiuto dell'Istituto per la Ricostruzione Industriale, che accordò un mutuo ventennale di 1.200.00o lire al tasso del 6,25\%. In effetti negli anni successivi la crisi venne superata, anche grazie al generale riassestamento delle economie mondiali, e gli Alinari stabilizzarono la loro attività su una linea di sostanziale continuità con il periodo precedente, ma con una quota crescente di attività commerciali ed editoriali.

Con una gestione molto accorta, anche se scarsamente innovativa, gli Alinari superarono con danni limitati la grave crisi della Seconda guerra mondiale; inoltre in virtù del forte processo inflattivo, la Società si trovò praticamente liberata dall'oneroso mutuo contratto con l'IRI.

In particolare, all'inizio degli anni Cinquanta la Alinari IDEA conobbe un periodo di forte espansione, come si vede dal grafico (Tab. 1).

\section{Tab. 1}

Capitale registrato da Alinari IDEA tra il 1949 e il 1956 in base all'utile lordo (in lire)

\begin{tabular}{|c|c|c|c|c|c|c|c|c|}
\hline 6.000 .000 & & & & & & & & \\
\hline \multicolumn{9}{|l|}{5.000 .000} \\
\hline \multicolumn{9}{|l|}{4.000 .000} \\
\hline \multicolumn{9}{|l|}{3.000 .000} \\
\hline \multicolumn{9}{|l|}{2.000 .000} \\
\hline \multicolumn{9}{|l|}{1.000 .000} \\
\hline & 1949 & 1950 & 1951 & 1952 & 1953 & 1954 & 1955 & 1956 \\
\hline
\end{tabular}

Un ruolo importante in questa ripresa di attività ebbe la parte commerciale basata in Italia, attraverso il sistema dei negozi. La Ditta infatti aveva da tempo stabilito eleganti negozi di vendita dei propri prodotti a Roma e a Napoli, ma anche a Firenze, dove pure era già attiva la sede centrale. Nel 1960, su un bilancio totale che pareggiava a 251.996.387 lire, con una parte commerciale che ammontava a 143.557.959 lire, 
le vendite dalla sede fiorentina (in massima parte per corrispondenza) assommavano a 87.660.871 lire, di cui 60.266.710 (69\%) in Italia e 27.394.161 (31\%) all'estero; mentre quelle dei tre negozi della Ditta (Roma, Napoli e Firenze) raggiungevano insieme la considerevole cifra di 55.897.088 lire, quasi pareggiando quindi le vendite della sede in Italia.

Questo dinamismo della gestione dei negozi corrispondeva però ad una relativa crisi del modello industriale classico tipico del periodo pre1920. Le vendite si indirizzavano soprattutto verso le riproduzioni di alta qualità, per formato e per tipo di stampa ${ }^{-19}$, mentre perdevano corrispondentemente peso le fotografie "piccole", cioè le stampe al bromuro d'argento in formato $18 \times 24$, destinate alle fototeche, agli istituti di istruzione e cultura, ai musei e agli studiosi, e tipiche del periodo precedente. Inoltre quella che perdeva di importanza era la quota delle vendite all'estero, assolutamente prevalente alle origini, e invece ora ridotta ad una porzione minoritaria.

Nel contempo si risparmiava sugli investimenti in macchinari e tecnologie per la riproduzione fotografica, ovvero su quello che era stato una volta il cuore produttivo dell'azienda, mentre si investiva invece per i negozi, per allestimenti e ammodernamenti, e perfino per l'acquisto di un appartamento per l'abitazione del nuovo gerente del negozio di Roma. Si seguiva per contro una politica di alti dividendi: nel 1955 vennero corrisposte agli azionisti 440 lire per ogni azione del valore nominale di 350 lire.

In definitiva tuttavia un "modello" di gestione che ancora riusciva a reggere, sia pure con un breve orizzonte in vista; la crisi venne dal contesto esterno e si pose come una crisi di crescita in cui si manifestarono i limiti endemici che avevano caratterizzato la gestione Ricasoli: cioè la scarsa capitalizzazione, con dividendi alti e ammortamenti e investimenti correnti bassi, la gestione conservativa dell'archivio e dell'apparato tecnico industriale dell'azienda senza affrontare sfide incombenti, soprattutto quella del colore; e il ricorso a indebitamento nei confronti di istituti di credito per investimenti o operazioni straordinarie.

\section{La crisi dei "fotografi editori" e l'intervento di Vittorio Cini fra impresa e mecenatismo (1958-1973)}

Se la Alinari IDEA riusciva a stare sul mercato distribuendo utili non inconsistenti agli azionisti, i tradizionali concorrenti, Anderson e Brogi, manifestavano sempre maggiori difficoltà a continuare l'attività. Dei contatti e dei pourparler erano intercorsi già da tempo, ma la prima proposta ufficiale di un acquisto di Anderson approdò nel CdA Alinari il 4 maggio 1957. Poco dopo contatti furono stabiliti anche con Brogi, sia pure su basi leggermente diverse ${ }^{-20}$.

L'offerta di Anderson era in realtà insieme allettante ed imbarazzante per la Alinari IDEA. Ricasoli e gli altri aristocratici toscani che amministravano l'Alinari IDEA non avevano la capacità o la volontà di trovare i capitali necessari (che pure equivalevano, come si vede dal 
grafico 1, all'ammontare di sole sei o sette annate dell'utile lordo registrato dall'azienda in quegli anni). Come recita il verbale del 4 maggio 1957:
Il consiglio prende atto con compiacimento di questa trattativa ed all'u- nanimità [...] delibera che la trattativa stessa sia proseguita, cercando di addivenire ad una intesa proficua per la Casa Alinari. II Consiglio precisa però che, non disponendo attualmente la Casa Alinari di capi- tali liquidi per eseguire tale acquisto, l'operazione possa essere perfe- zionata solo dopo che saranno stati assiemati i capitali occorrenti ${ }^{21}$.

In questa ricerca di nuovi capitali Ricasoli si rivolse a Vittorio Cini, ma questi, mentre da una parte manifestò un vivo interesse per la proposta, si dichiarò disponibile solo per un'acquisizione in toto dell'intero pacchetto azionario.

Di fronte a questo atteggiamento molto disponibile (Cini pagò un prezzo molto alto per il pacchetto di azioni di Ricasoli) ma anche molto fermo, il barone fiorentino fece un passo indietro, e alla fine del 1957 prese la parola in sede di CdA
per comunicare al Consiglio ed al Collegio sindacale di aver ceduto, pur con rammarico, il pacchetto di maggioranza delle proprie azioni I.D.E.A. al Conte Senatore Vittorio Cini ${ }^{22}$.

Anche nel caso di Cini non è possibile determinare con precisione, allo stato attuale della ricerca, quali fossero le motivazioni della scelta. Considerando però alcuni aspetti della personalità di Cini ${ }^{-23}$, all'epoca uno dei più forti e potenti esponenti della finanza italiana, già impegnato - specie dopo la morte, nel 1949, del figlio Giorgio - in una importante opera di mecenatismo culturale, l'acquisizione di Alinari poteva apparire oltre e forse più che una scelta imprenditoriale, una operazione di politica culturale, tesa a salvaguardare e valorizzare un patrimonio "nazionale", lungo una linea che non era certo estranea agli obiettivi del "gruppo veneziano" di cui era stato esponente di spicco, né alla sua precedente attività di Commissario dell'E42.

$\mathrm{Si}$ può capire quindi che Cini fosse fermamente intenzionato ad acquisire non solo il pacchetto di maggioranza della Alinari IDEA, ma tutto il pacchetto e quindi l'intero patrimonio, con l'intento poi di accrescerlo con i fondi Anderson e Brogi.

Questo fu quanto accadde nel corso del 1958 e agli inizi del 1959: a dicembre del 1958 si deliberò l'aumento del capitale sociale da $£$ 2.450.000 a $£$ 35.000.000 (poi aumentato ancora l'anno successivo a $£$ 90.000.000), motivato esplicitamente con la necessità delle nuove acquisizioni (Anderson e Brogi, ma non solo) ${ }^{-24}$.

In immediata successione di tempo Cini fece acquisire i patrimoni fotografici di Anderson e Brogi. Dalla documentazione disponibile sembra che Cini non avesse in realtà un orientamento troppo preciso circa il 
destino della Alinari IDEA. Sicuramente aveva fin dall'inizio escluso di trasferire il patrimonio fotografico a Venezia presso la Fondazione Cini, ed oltre a nominare Federico Gentile nel CdA, attraverso di lui aveva stabilito un rapporto molto forte di Alinari IDEA con l'Istituto per la Collaborazione Culturale (diretto da Gentile ed editore fra l'altro della Enciclopedia Universale dell'Arte), il che sembra mostrare che inizialmente vi fosse una strategia imprenditoriale innovativa nel settore editoriale ${ }^{-25}$; in seguito però le azioni furono trasferite alla Fondazione Cini, e apparve chiaro l'intento di consolidamento patrimoniale, anche, e forse soprattutto, a fini culturali più che imprenditoriali.

Sarebbe troppo lungo esaminare qui i meccanismi piuttosto complessi ed elaborati messi in atto per regolare i rapporti fra la Fondazione Cini e la Alinari IDEA ${ }^{-26}$, ma si può dire che in sostanza in questa fase IDEA consolidò ampiamente il patrimonio, incorporando anche altri fondi e archivi fotografici di notevole valore, come quelli di Chauffourier e Fiorentini, e sviluppando anche nuove campagne di ripresa nel Veneto ${ }^{-27}$. Nonostante questo forte aumento del patrimonio fotografico, l'azienda mantenne sostanzialmente lo stile di amministrazione stabilito nel periodo Ricasoli, come testimoniato anche dalla permanenza di Agostinelli nella carica di direttore, e dal fatto che ancora nel $1970 \mathrm{i}$ negozi mantenevano il loro ruolo molto importante nelle entrate dell'azienda ${ }^{-28}$; la sola novità rilevante e confortante era il forte aumento in valore delle vendite all'estero dalla sede, che giungevano quasi a pareggiare le vendite in Italia.

In realtà, queste cifre apparentemente rassicuranti nascondevano alcuni forti elementi di crisi. La conduzione Cini se era intervenuta fortemente nella capitalizzazione e nelle acquisizioni, non aveva però, specie nella fase in cui le azioni erano depositate presso la Fondazione, svolto nessun reale intervento circa la conduzione tecnica dell'IDEA. Il punto di svolta avvenne attorno ad un progetto di radicale trasformazione tecnico-produttiva della Alinari IDEA, che prevedeva di abbandonare la sede storica nel centro di Firenze, per acquisire uno stabilimento nella zona industriale, dotato di macchinari avanzati e finalizzato a una produzione rinnovata appunto sulla base del colore (divenuto una questione ineludibile), il che avrebbe comportato a sua volta la necessità di nuove campagne di ripresa ${ }^{29}$.

Di fronte a questo progetto così impegnativo, la Direzione della Fondazione Cini espresse tutte le sue perplessità, dichiarando esplicitamente al Senatore Cini che non rientrava nelle finalità della Fondazione, come ente culturale, questa iniziativa spiccatamente imprenditoriale della Alinari IDEA ${ }^{-30}$. Cini prese atto della situazione, avocò nuovamente a sé le azioni Alinari, che erano state attribuite alla Fondazione Cini, e per qualche tempo si occupò di un possibile sviluppo dell'azienda. Ormai però Cini, che aveva oltrepassato gli 85 anni, non aveva probabilmente l'energia per cimentarsi in un'impresa oggettivamente molto difficile e impegnativa. Allo stato attuale della ricerca non abbiamo reperito documentazione più probante, ma probabilmente 
è per questi motivi che Cini decise nel 1973 di cedere il suo pacchetto azionario a Renato Zevi, imprenditore milanese, persona colta, collezionista, già fra i principali finanziatori del "Piccolo" di Milano.

\section{Mercato e cultura: dalla fotografia come documentazione del patri- monio alla valorizzazione della fotografia come patrimonio (1973- 1982)}

La proprietà Zevi inaugurò una stagione radicalmente nuova nella conduzione della Alinari IDEA, ma non sulla linea di rinnovamento imprenditoriale che era stata prospettata negli ultimi anni della proprietà Cini.

La nuova linea aziendale fu condizionata da un evento nuovo, quasi sicuramente non previsto al momento dell'acquisizione dell'azienda, cioè la grande mostra del 1977 al Forte Belvedere (Gli Alinari fotografi a Firenze 1852-1920) ${ }^{-31}$, frutto di scelte culturali interne ma anche del clima politico-culturale che era seguito dopo le elezioni amministrative del 1975 .

Il progetto della mostra era molto interessante e aperto per l'epoca. Contemplava infatti una parte specifica sul nucleo fondamentale del lavoro degli Alinari, affidata a tre giovani e validissimi storici dell'arte; ma questa sezione era preceduta e seguita da altre parti relative al contesto urbano e sociale di Firenze ("la loro città, la gente, le strade, il lavoro"), al ritratto, alle altre città italiane e al paesaggio ${ }^{-32}$.

La scelta, vista retrospettivamente dal punto di vista che ci interessa, cioè del patrimonio fotografico Alinari, era abbastanza singolare, ma molto significativa. Infatti, si trascurava assolutamente tutto quello che era stato acquisito durante la proprietà Cini, in termini di archivi fotografici dei grandi competitori storici degli Alinari, e si restringeva il campo sostanzialmente all'attività non solo della Alinari come ditta, ma in realtà al solo periodo aureo, quello in cui era ancora azienda a conduzione familiare, fra la metà dell'Ottocento e il primo dopoguerra.

Per contro, si valorizzava in maniera del tutto nuova e inusitata una parte dell'archivio che era stata in precedenza assolutamente negletta, quella relativa al cosiddetto "patronato". Si trattava delle fotografie che erano state prodotte dagli Alinari su committenza esterna, molto spesso ritratti, ma anche foto di ambienti, di case, di negozi, di opifici, di istituti, i quali potevano avere commissionato agli Alinari una riproduzione fotografica per i più svariati motivi, e di cui gli Alinari stessi avevano conservato spesso il negativo. Si trattava di un tipo di lavorazione già secondario nell'economia della ditta, e addirittura quasi abbandonato dalla conduzione Ricasoli in poi.

Dopo un lungo e complesso lavoro di recupero e di riordinamento, questa parte dell'archivio fornì la base per le due sezioni che risultarono fra le più "popolari” della mostra: quella storico-sociale e quella del ritratto.

La mostra di Forte Belvedere incontrò un eccezionale successo, non preventivato in tali dimensioni neppure dalle più ottimistiche previsioni 
degli organizzatori (600.000 visitatori paganti e oltre 100.000 copie dell'importante catalogo vendute). Questo evento fu particolarmente importante per gli Alinari e non solo, dato che segnalò un cambiamento nel gusto comune verso la fotografia d'epoca, e più in generale verso la fotografia come patrimonio culturale. I consumi di fotografia avevano avuto una netta impennata in quegli anni grazie alla diffusione di tecnologie molto più accessibili e aperte al consumo di massa- ${ }^{33}$; ma il riconoscimento sociale della fotografia come patrimonio culturale era molto limitato.

Sul piano pratico si verificò negli anni successivi una intensificazione assolutamente rapida ed amplissima dell'interesse per la fotografia d'epoca, testimoniata dalla frequenza e articolazione a tutti i livelli delle mostre e delle pubblicazioni ${ }^{-{ }^{34}}$. Si deve inoltre tenere presente che in quel periodo, accanto alle iniziative e alle opere più importanti e significative, stava letteralmente esplodendo una editoria a carattere divulgativo ed evocativo, con scarso o nullo apparato critico e inquadramento storico, ma non di rado in grado di riportare alla luce fondi di notevole interesse documentativo ${ }^{-35}$.

Si trattava di una esplosione che si sarebbe probabilmente verificata in altre forme in ogni caso; ma sicuramente il successo della mostra Alinari stabilì un riferimento molto importante per gran parte degli sviluppi successivi, portando all'attenzione del grande pubblico il ruolo memoriale, evocativo, della fotografia come macchina della memoria per il recupero del passato.

Il periodo della conduzione Zevi rappresenta quindi, dal lato del patrimonio (anche nella sua accezione economica), una forte innovazione metodologica, nel senso che il focus operativo passa da una considerazione della fotografia come strumento funzionale alla documentazione del patrimonio architettonico, artistico e paesaggistico, a una considerazione della fotografia come patrimonio essa stessa. In un certo senso, dal punto di vista imprenditoriale, si lascia in secondo piano l'idea di riorganizzare o rimodernare l'attività di documentazione fotografica in forme nuove, e si passa all'idea di utilizzare il patrimonio fotografico già accumulato per il suo valore storico e memoriale, più che per il suo valore funzionale alla tradizionale attività di documentazione tipica dei decenni precedenti.

Per quanto molti degli usi tipici delle fotografie Alinari perdurassero per un certo tempo (dall'uso più classico e diffuso nei manuali di storia dell'arte, a quelli più occasionali ma non meno significativi di larga diffusione, ad esempio nelle carrozze ferroviarie o negli "intervalli" televisivi) ormai il secondo periodo della gestione Zevi fu indirizzato soprattutto alla individuazione di un nuovo modello economico per mettere a frutto il patrimonio dopo la fiammata rapidissima ma anche effimera della richiesta immediatamente successiva alla grande mostra del 1977. Una delle strade che furono percorse fu sicuramente quella dell'editoria di pregio, una realtà che era sempre stata presente nelle strategie della ditta, con pubblicazioni come la Biblioteca di disegni, 28 volumi 
in 325 esemplari nella tradizionale e pregiata lavorazione in collotipia, così come quella della vendita delle riproduzioni, nonché di esposizioni ulteriori sulla traccia della fortunata iniziativa del 1977. Fu tentata anche la strada di una editoria direttamente collegata alla mostra del 1977, adattata alle varie città o località italiane. Un'altra strada percorsa fu quella, per l'epoca innovativa, della diffusione del patrimonio visuale dei tre grandi fotografi editori attraverso un'edizione in microfiche.

Si trattava però di un compito molto arduo. Per motivi su cui gli studi e la documentazione disponibile non permettono un'analisi dettagliata e sicura, l'azienda entrò in crisi economica, al punto da essere costretta a vendere una parte dell'immobile. Si prospettò una possibile acquisizione pubblica da parte della Regione Toscana, che però non ebbe esito, e alla fine di un breve periodo di incertezza ${ }^{-36}$, si ebbe il passaggio di proprietà dell'azienda, che portò alla Presidenza Claudio de Polo Saibanti, all'epoca amministratore di una grande azienda di rilievo nazionale, con sede a Trieste.

\section{Mercato e cultura: la competizione su scala mondiale per il controllo della memoria visuale nella civiltà dell'immagine (1982-2019)}

La conduzione di Claudio de Polo Saibanti, dopo una prima fase un po' incerta, si attestò su una linea che implicò una serie di elementi di novità che permettono di parlarne come di una fase nuova che trasformò profondamente non solo lo stile di attività dell'azienda, ma anche e soprattutto la consistenza e la caratterizzazione del patrimonio.

L'Alinari IDEA di de Polo riprese in un primo momento su nuove basi l'elemento innovativo della gestione Zevi, cioè la forte valorizzazione dell'immagine pubblica dell'azienda e del nome Alinari. Inaugurò uno spazio espositivo in una delle vie privilegiate della zona del lusso del centro storico di Firenze, cercando di svolgere un'attività di qualità e di prestigio, ma presto si rivolse nella direzione di un accrescimento ulteriore del patrimonio lungo una linea di acquisizioni a largo raggio di archivi e stampe fotografiche d'epoca, su scala nazionale e poi presto anche su scala internazionale.

Questo tipo di sviluppo fu favorito dal fatto che i fondi e gli archivi fotografici ancora non godevano di una considerazione adeguata; in molti casi archivi interi e intere collezioni private potevano essere ceduti a prezzi molto bassi o addirittura gratuitamente. Non esisteva una politica di acquisizione coordinata e centralizzata da parte degli organi centrali dello Stato; gli enti pubblici territoriali si muovevano a volte attivamente e con intelligenza, ma con logiche molto diverse, sottoposte comunque a forti limiti economici e burocratici. In queste condizioni, la Alinari IDEA sviluppò una politica di acquisizioni estesa e a suo modo sistematica, condotta direttamente dal nuovo proprietario, sia pure con la consulenza e l'aiuto di una serie di esperti. Claudio de Polo, anch'egli persona colta, collezionista bibliofilo, proveniente da Trieste, dove aveva un ruolo di rilievo negli ambienti industriali e finanziari della città, aveva conosciuto e frequentato il Sen. Cini e si concepiva come 
un continuatore della sua opera di incremento delle collezioni Alinari e di valorizzazione della ditta, anche in funzione del ruolo storico di Alinari nella cultura nazionale. De Polo svolse questa attività però in forme molto diverse da quelle di Cini. Mentre il senatore aveva puntato soprattutto sul ricongiungimento, per così dire, presso gli Alinari degli archivi fotografici dei maggiori fotografi editori italiani, de Polo aprì la via di una raccolta estesa alle più varie forme di fotografia, non solo d'epoca e non solo di documentazione del patrimonio artistico.

Protrattasi con varie fasi, fino agli ultimi anni di attività dell'azienda, questa vasta opera di acquisizione è quella che ha portato, nel corso degli ultimi quattro decenni, il patrimonio degli Alinari da circa 300.000 "pezzi" della conduzione Cini agli oltre 5.000.000 attuali.

La cifra "collezionistica" impressa dal nuovo proprietario era evidente non solo nella varietà assoluta di materiali raccolti (dalle fotografie vere e proprie, negative e positive, agli album, alle pubblicazioni fotografiche, all'oggettistica, ecc.) ma anche nella composizione delle singole sezioni.

Sarebbe troppo lungo elencare anche sommariamente qui i fondi acquisiti, o sul mercato, o attraverso donazioni; basti dire che vanno da una collezione di dagherrotipi molto cospicua, ad una serie importante di fondi di autori ed enti italiani (che costituiscono senz'altro il nucleo fondamentale), ad un fondo di album di eccezionale rilievo anche a livello internazionale, e a un certo numero di fotografie di autori stranieri, in parte acquisite a suo tempo anche come pezzi individuali per l'allestimento del Museo Nazionale Alinari della Fotografia ${ }^{37}$.

Un altro aspetto chiave della gestione de Polo fu che dette forte impulso all'attività espositiva, editoriale, culturale in senso lato legata alla fotografia, a livello nazionale e internazionale.

L'attività espositiva, iniziata già dai primi anni, si estese e si specializzò, trovando spazi innanzitutto in quelli direttamente controllati da Alinari, dapprima nella piccola sede in Palazzo Rucellai in via della Vigna Nuova, e poi in Piazza Santa Maria Novella, dove dal 2006 al 2014 viene allestito il Museo Nazionale Alinari della Fotografia, con una parte museale fissa e diversi ampi locali adibiti a mostre temporanee; ma numerose furono anche le iniziative in sedi esterne, in Italia e anche all'estero ${ }^{-38}$.

In collegamento con tale attività espositiva, venne realizzata una intensa attività editoriale, dato che sistematicamente si cercava di abbinare all'esposizione un catalogo, molto spesso pubblicato in proprio da Alinari, o in collaborazione con altre case editrici. La qualità delle mostre e dei relativi cataloghi è stata di regola molto alta, con un amplissimo range di autori, allestitori, collaboratori coinvolti e con una notevole cura degli allestimenti e delle edizioni.

Occorre sottolineare che in progresso di tempo si è formato presso Alinari un nucleo rinnovato di operatori e operatrici molto qualificati, con livelli di istruzione di partenza spesso molto elevati, ulteriormente perfezionati nel lavoro in azienda, in modo da realizzare un insieme di 
competenze e di specializzazioni veramente rilevante a livello nazionale e internazionale.

Oltre a questa attività editoriale va ricordata, fin dal 1985, la pubblicazione di una rivista, all'epoca esperienza piuttosto innovativa, con il titolo di "Fotologia", diretta da Italo Zannier.

Inoltre, va ricordata la risistemazione e la cura della Biblioteca, indubbiamente una delle più ricche e originali in Italia sul tema, per il fondo storico, che contiene molti volumi rarissimi e importanti, ed anche per le acquisizioni successive nel tempo di biblioteche personali di studiosi ed esperti.

Notevoli anche le attività collegate alla formazione e al restauro, con la realizzazione di un laboratorio interno, e con varie attività formative, fra cui tirocini universitari e corsi specializzati, come quello in collaborazione con l'Opificio delle Pietre Dure di Firenze.

Infine, oltre alle acquisizioni che portarono all'aumento imponente del patrimonio fotografico, va ricordato il fatto che dagli ultimi anni del XX secolo Alinari si impegnò nell'attività di digitalizzazione delle proprie fotografie. Questa attività, che nelle intenzioni dell'azienda doveva realizzare un cespite importante di entrate, si è sviluppata nel tempo, giungendo a realizzare la digitalizzazione di una parte significativa del patrimonio complessivo, o di altri fondi gestiti, per un totale stimato in oltre 200.00o immagini. Cosa particolarmente interessante nel panorama della fotografia italiana, una quantità molto rilevante di fondi e archivi fotografici di proprietà di terzi sono stati affidati in gestione, per la parte di diffusione e commercializzazione attraverso canali digitali, da vari enti pubblici e privati (fra i quali Touring Club Italiano, Istituto Luce, Archivio Ansaldo, Teche Rai, Ansa, Folco Quilici, Fosco Maraini, ecc.) ad Alinari, al punto che la ditta dichiarava già attorno al 2008 di gestire in tutto, fra patrimonio proprio e patrimonio gestito, oltre 40 milioni di immagini: una cifra nettamente inferiore, ma pur sempre dello stesso ordine di grandezza rispetto a quelli che erano allora i giganti mondiali del settore, come Corbis o Getty Images ${ }^{39}$.

Alinari non si poteva paragonare ai due giganti della stock photography, però aveva già iniziato un'attività di raccolta di fondi fotografici, che su scala italiana era abbastanza sistematica ed estesa, e si era mossa tempestivamente anche sul terreno delle tecnologie digitali, per cui si può dire che rientrasse in quella serie di aziende di media dimensione che per un certo periodo hanno accompagnato la crescita di mercato del digitale, salvo poi entrare in crisi in seguito alla recessione del 2008-2013-40.

Da un punto di vista storico-economico è impossibile al momento ricostruire con precisione gli eventi che hanno portato alla crisi dell'azienda. Alcune tappe evidenti possono essere comunque ricordate. Nel 2007, di fronte ad una forte crisi di crescita, in dipendenza delle opportunità, ma anche delle necessità di investimento portate dal nuovo mercato digitale, Alinari creò una joint venture con uno dei più autorevoli gruppi italiani, ovvero con il Gruppo "Sole24ore”, organo di Confindustria, con 
l'intento di consolidare e rilanciare il proprio ruolo nella competizione in atto sul mercato, come uno "tra i pochi grandi players mondiali nelle immagini di storia, cultura e industria e nelle foto d'arte”. In realtà il matrimonio con il "Sole 24ore", maturato nel momento in cui quest'ultimo si quotava in borsa, subì le conseguenze della crisi del quotidiano di Confindustria, e in genere del rallentamento dei mercati dopo il 2008, al punto che la joint venture fu liquidata nel $2012^{-41}$.

Per quanto la chiusura della joint venture non avesse in sé determinato un danno patrimoniale all'azienda, certamente si ridimensionava drasticamente una prospettiva di sviluppo su cui la proprietà della ditta aveva puntato molto, senza che vi fossero le basi sostitutive per un rilancio interno $-{ }^{42}$.

Nel 2014, per la necessità di lavori di adeguamento dei locali di proprietà del Comune di Firenze, dovette chiudere il MNAF, e negli anni successivi non si ebbe la forza di riaprirlo. Neppure ebbe successo sul piano economico il pur interessante tentativo di realizzare a Trieste presso il Castello di San Giusto un Alinari Image Museum, basato tutto sui contenuti digitali, inaugurato nel 2016, ma chiuso definitivamente nell'aprile 2019. Si giunse così, nonostante alcuni tentativi di stabilire accordi $o$ attività finalizzate a ottenere finanziamenti su parti di patrimonio, alla decisione, fra il 2018 e il 2019, di alienare sia il palazzo storico di Largo Alinari, sia il patrimonio fotografico.

\section{Conclusioni}

Il patrimonio fotografico Alinari, alla luce di questo excursus storico, è sicuramente un patrimonio di eccezionale interesse, non solo per il contesto italiano, ma anche per quello internazionale. Appare evidente che nel corso dei quasi 170 anni di vita, questo patrimonio non si è semplicemente accumulato, ma si è stratificato secondo le varie e molto diverse fasi di vita dell'azienda che lo ha prodotto o acquisito. Quindi il patrimonio oggi appare composto da vari nuclei molto diversi fra loro, sia per consistenza e materiali, ma soprattutto per il loro significato e per la loro destinazione in relazione alle varie fasi di vita dell'azienda (si pensi ai musealia e alla parte digitalizzata), il che rende complessa e non facile quella riorganizzazione di cui si parlava all'inizio. Inoltre il passaggio attuale, che porta per la prima volta alla proprietà pubblica (anche se in diverse altre fasi, come abbiamo visto, interventi della mano pubblica o di proprietari che abbinavano una forte propensione al mecenatismo alla dimensione imprenditoriale non erano mancati) comporta un notevole elemento di discontinuità. Questa discontinuità in parte discende dal fatto, molto interessante per gli studiosi, che la gestione del patrimonio sarà meno condizionata dalle preoccupazioni degli andamenti del mercato e molto più dall'esigenza di promuovere, oltre che la conservazione e adeguata riorganizzazione del patrimonio, la pubblica fruizione e l'utilizzazione del patrimonio in campo educativo, di studio e di ricerca, ma anche gli usi che incentivino la fruizione partecipata e diffusa di pubblici diversi e anche non specializzati. 
Si tratta di una sfida molto importante e non facile per tutti coloro che hanno a cuore il patrimonio fotografico italiano. La Società Italiana per lo Studio della Fotografia, che ha seguito con molta attenzione le fasi che hanno portato all'acquisizione, ha ricevuto l'incarico di predisporre uno studio sulle radici storiche del patrimonio Alinari, di cui questo saggio costituisce un'anticipazione, ed inoltre un'attività tesa alla consultazione di possibili interlocutori sul piano scientifico e culturale per la futura attività della Fondazione. Cercherà di svolgere questo ruolo interessando, per quanto possibile, tutta la comunità di studiosi e di operatori culturali in campo fotografico a partecipare con contributi di idee e di proposte all'attività di questo nuovo polo pubblico che, per la sua importanza storica e per la qualità e quantità del patrimonio posseduto, può svolgere un ruolo di grande rilievo. Molto dipenderà però dalla collaborazione e dalla capacità di ideazione e di iniziativa degli operatori, dei fotografi, degli studiosi, dei curatori, degli educatori, di fronte a questa opportunità.

- ${ }^{1}$ Bollettino Ufficiale 2019.

$-{ }^{2}$ Esistono molti studi importanti e approfonditi, in particolare sul periodo aureo dell'azienda familiare (1852-1920) e quindi sul fondo fotografico relativo, che è in realtà solo una parte dell'attuale patrimonio Alinari. Qui ci limitiamo a citare Quintavalle 2003; cfr. inoltre Quintavalle / Maffioli 2003 con testi di vari autori italiani e stranieri.

$-{ }^{3}$ Cfr. Atti inchiesta industriale 1873-74a. Dalla stessa fonte anche le successive citazioni nel testo.

$-{ }^{4}$ Atti inchiesta industriale 1873-74b.

$-{ }^{5}$ Anderson dichiarò apertamente, nella stessa Inchiesta, che a suo parere la vendita in stock ai corrispondenti esteri non aveva futuro, e di preferire la vendita ai turisti che affluivano in Italia ormai in numero sempre rapidamente crescente.
$-{ }^{6}$ Cfr. Tomassini 1987, p. 65. Brogi in quell'occasione auspicò la costituzione di una "Unione fra $i$ fotografi editori", che non ebbe poi attuazione, ma che si può considerare un'anticipazione di quello che fu poi il 'trust' del 1904 con Alinari e Anderson. $-{ }^{7}$ Fondata nel 1889 , nel cinquantenario dell'invenzione della fotografia, la SFI riuniva su base nazionale le diverse anime della cultura fotografica presenti in Italia, con adesioni di eccezionale prestigio, come quella del principe ereditario, poi Re d'Italia, Vittorio Emanuele III. Cfr. Caputo Calloud 1992.

$-{ }^{8}$ Cfr. Sisi 2012.

$-{ }^{9}$ Cfr. Tomassini 2013, p. 302.

- ${ }^{10}$ Quintavalle 2003, p.

420.

- ${ }^{11}$ Sarebbe interessante analizzare attraverso la verifica dell'effettiva diffusione, certamente molto larga, di questa parte del patrimonio, quanto esso abbia contribuito a creare un'immagine antropologica, occidentalizzata, dell'Italia nel suo complesso; sia nel senso della formazione antropologica dell'Italia contemporanea, del suo spirito, del suo immaginario, della sua costruzione identitaria, per se stessa e per gli stranieri; sia infine nel senso basilare della documentazione dei mondi e degli specifici etnici. Sotto questo aspetto, secondo alcune indicazioni metodologiche che debbo a Francesco Faeta, Alinari si potrebbe considerare anche come un importante giacimento di immagini etnografiche relative al nostro Paese; ed anche uno dei più interessanti performatori dell'identità nazionale attraverso la stretta messa in relazione dell'immagine della bellezza artistica e della complementare e antagonistica presenza 
dei mondi popolari e degli specifici etnici. Va inoltre considerata la possibile contestualizzazione rispetto sia ai tentativi di Santoponte di realizzare una specie di inventario visivo anche di tipo storico sociale, sia anche all'originale filosofia di Giovanni Gargiolli e del Gabinetto Fotografico Nazionale, permettendo in questo modo di affiancare ancora meglio la prospettiva storico-sociale e quella storico-artistica nella considerazione del patrimonio dei fotografi editori.

- ${ }^{12}$ Cfr. Alinari 1906;

Alinari 1908; Alinari 1921. - ${ }^{13}$ Cfr. La serrata 1915.

- ${ }^{14}$ Cfr. Ciuffoletti / Sesti 2003, p. 240.

- ${ }^{15}$ Cfr. per questi aspetti ibid.; ringrazio inoltre Emanuela Sesti per avermi anticipato la bozza di un suo saggio dal titolo La fotografia d'arte dei Fratelli Alinari dal 1852 ad oggi e gli archivi fotografici entrati nelle collezioni Alinari al tempo di Vittorio Cini, in corso di pubblicazione negli atti della giornata di studi Per un archivio fotografico dell'arte italiana. Vittorio Cini, la Fondazione Giorgio Cini e la Fratelli Alinari, Venezia, Fondazione Giorgio Cini, 5 giugno 2018.

$-{ }^{16}$ \#ASAI 1929

Nella stessa seduta si decideva di provvedere al "rinnovamento delle collezioni fotografiche", ma data la situazione solo per Firenze e per la Toscana, e "limitatamente alle esigenze del lavoro di commissione".

- 17 Ivi, Seduta del

8 febbraio 1932. Nel

frattempo, infatti, l'Istituto

L.U.C.E., che aveva assorbito il Gabinetto Fotografico Nazionale, stava intraprendendo un'azione di diffusione e vendita di fotografie del patrimonio culturale italiano.

- 18 Ibid. Nel 1932 si registrano perdite per $\mathbf{5 4 . 0 0 0}$ lire, dopo aver assorbito tutti gli utili degli anni precedenti. Questo comportò la dismissione in particolare della sala di posa e della parte di lavorazioni dedicate al ritratto, qualificate come non più redditizie.

$-{ }^{19}$ In particolare le fotocollotipie, le foto dirette a colori e i disegni dei grandi maestri, che complessivamente nel 1960 formavano ancora oltre il $60 \%$ del valore del magazzino. Tutti i dati in questa parte del testo sono elaborazioni dalla serie dei bilanci annuali conservati in ASAI.

-20 Per Brogi infatti

si trattava di cedere

l'archivio delle lastre 'storiche', mentre l'attività dello studio fotografico sarebbe stata continuata da Laurati, genero di Brogi e fotografo egli stesso.

Anderson invece intendeva vendere tutto il patrimonio di immagini e tutto il pacchetto azionario della ditta.

- 21 \#ASAI 1957.

- 22 Ivi, Seduta del 27

dicembre 1957.

-23 Senatore e

commissario per l'Expo '42, fu anche ministro nel 1943. Non esitò nel contempo a prendere posizioni anche apertamente critiche nei confronti di Mussolini, ancor prima del 25 luglio 1943. Internato per qualche tempo a Dachau, da dove riusci a fuggire in Svizzera, fu riabilitato dopo la fine della guerra dalle accuse di collaborazione con il fascismo (cfr. Reberschak 1981).

- 24\#ASAI 1958.

L'aumento di capitale avvenne mediante emissione di 93.000 nuove azioni da 350 lire nominali, offerte in opzione agli azionisti in ragione di 93 azioni contro 7 vecchie. -25 Nel 1960 in

effetti I'Istituto per la Collaborazione Culturale appariva come il maggiore cliente italiano di Alinari, con 1.210.000 lire, superando il Ministero degli Esteri che occupava il secondo posto (cfr. \#ASAI 1960).

$-{ }^{26}$ Per un

approfondimento cfr.

Emanuela Sesti, La

fotografia d'arte dei Fratelli

Alinari, cit.

- 27 Ibid.; cfr. anche Alinari 1963.

- 28 Anzi, lo

incrementavano

leggermente, passando da un incasso complessivo di 55.897.088 lire nel 1960, pari al $39 \%$ delle vendite totali, ad un incasso di $\mathbf{8 7 . 9 6 5 . 1 3 0 ~ l i r e ~ n e l ~} 1970$, pari al $41 \%$ del totale (dati ricavati da ASAI, serie Bilanci, agli anni corrispondenti).

- ${ }^{29}$ La documentazione di questi progetti e delle interlocuzioni fra Cini e i suoi stretti collaboratori e la Fondazione è in \#Archivio Cini.

-30 Ibid.

$-{ }^{31}$ Cfr. Settimelli / Zevi

1977.

- 32 Cfr. Ferretti / Conti / Spalletti 2003. Le sezioni sulla città e sul ritratto erano curate da Arnaldo Salvestrini, Fernando Tempesti, Lorenza Trionfi Honorati e Luigi Tomassini; la sezione Le altre città $e$ 
il paesaggio italiano era a cura di Filippo Zevi.

- ${ }^{33}$ Mi permetto di

rimandare a Tomassini

2018.

-34 Oltre alle grandi mostre del 1979, Fotografia italiana dell'Ottocento

e Fotografia pittorica 1889/1911 (cfr. in proposito Cavanna 2020, cap. 4), si andavano infatti pubblicando opere fondamentali per l'inquadramento storico e teorico dello studio della fotografia. Fra di esse, citiamo solo per brevità Bertelli / Bollati 1979; Miraglia 1981; e le traduzioni in italiano delle opere di Susan Sontag, Aaron Scharf e Roland Barthes, nonché il fortunato manuale di Zannier 1982.

- 35 Sono un indicatore di questo fenomeno l'aumento del numero delle pubblicazioni specializzate (per il caso italiano, la più completa è quella annessa al volume Cavanna 2020 accessibile con distribuzione Creative Commons in <http:// www.pierangelocavanna. it/2020/05/22/

il-miele-e-largentobibliografia-2020/> 07.09.2020); ma in realtà il fenomeno è stato molto più ampio, e riguardava non solo pubblicazioni a volte molto periferiche e locali, non sempre registrate nei repertori bibliografici, ma anche in forme diverse da quella del libro, come gli inserti - molto popolari - nei maggiori quotidiani nazionali. Inoltre vi è una ricerca tutta da fare sulle iniziative di costituzione di archivi fotografici su scala territoriale, presso enti locali, biblioteche, archivi, musei, centri di documentazione e studio, che fornirono un impulso fondamentale, e spesso qualitativamente elevato, alla diffusione di una cultura fotografica e al recupero di fondi e archivi fotografici.

- 36 Durante questo periodo la Regione Toscana si dotò di un organo prevalentemente scientifico e inizialmente solo in parte operativo, con il nome di Archivio Fotografico Toscano (AFT), la cui sede fu stabilita a Prato, ma con finanziamenti e compiti regionali. L'AFT, già attivo dal 1979, fu aperto al pubblico nel $1985 \mathrm{e}$ pubblicò dal 1985 al 2009 una rivista di notevole rilievo nel panorama italiano, oggi disponibile in versione digitale in <http://rivista.aft.it/aftriv/ controller> (07.09.2020). - 37 Una delle caratteristiche di questa parte dell'archivio è che comprende raccolte $e$ fondi acquistati o donati da alcuni dei maggiori collezionisti-studiosi italiani, come Daniela Palazzoli, Italo Zannier, Dario Reteuna, Michele Falzone del Barbarò, Piero Becchetti, ed altri, ed anche da alcuni stranieri come Christophe Blatt e Henri Favrod. Oltre a ciò, contiene archivi di numerosi fotografi italiani, alcuni fondi di fotografi stranieri attivi in Italia come Von Gloeden, fondi provenienti da enti, istituzioni e imprese, nonché alcuni fondi di agenzie fotografiche o di periodici italiani e stranieri. - 38 In particolare in

Francia si possono ricordare la mostra organizzata nel 2004 al Pavillon des Arts di Parigi con il titolo $\mathrm{Vu}$ d'Italie 1841-1941, la prima che presentasse complessivamente la fotografia italiana in Francia, nonché diverse altre mostre tematiche presso altri istituti, come il Petit Palais e I'Istituto

Italiano di Cultura di Parigi. - ${ }^{39}$ Che allora

dichiaravano

rispettivamente di possedere rispettivamente 60 e 90 milioni di immagini. Per un inquadramento generale della evoluzione delle grandi agenzie di stock, in primo luogo Getty Images, si veda Frosh 2003. Per un quadro più aggiornato, che tiene conto dei più recenti sviluppi che coinvolgono oltre a Corbis e Getty anche altri attori attualmente di primo piano nel web, come Flickr, cfr.

Runge 2020.

- 40 Secondo

Glückler / Panitz 2013

(p. 9), la crisi finanziaria del 2008 aveva colpito

il settore e favorito la concentrazione: le quattro maggiori agenzie di stock, cioè Getty, Corbis, Shutterstock e Fotolia coprivano nel 2011 circa la metà degli incassi di tutto il settore.

$-{ }^{41}$ Cfr. ad esempio, Finito

il matrimonio 2012.

- 42 Ibid. In quello stesso anno 2012 , il bilancio della ditta fiorentina esibiva infatti un utile, sia pure limitato a $\mathbf{2 4 . 0 0 0}$ euro, ma a fronte di un complesso di ricavi di soli 560.000 euro e un patrimonio netto di 7,5 milioni, assolutamente fuori scala rispetto alle necessità di investimento per tenere il passo dei maggiori competitori sul mercato internazionale. 
Alinari 1906 Alinari, Dresda. Riproduzioni pubblicate per cura di Vittorio Alinari, proprietario dello stabilimento fotografico Fratelli Alinari, Firenze, Barbera, 1906.

Alinari 1908 Alinari, Paris. Photographies de la Maison Alinari Frères Florence, Firenze, Barbera, 1908.

Alinari 1921 Alinari, La Grèce. Photographies de la maison Alinari Frères, reproduction ipsographique de l'edition 1908, Firenze, Fratelli Alinari Società Anonima, Istituto di Edizioni Artistiche I.D.E.A, 1921.

Alinari 1963 Alinari 1963 Alinari, Catalogo delle fotografie Alinari del Veneto, Firenze, Alinari, 1963.

Atti inchiesta industriale 1873-74a Atti del Comitato dell'inchiesta industriale, vol. II, Deposizioni orali, categoria 13-2 (incisione, litografie e fotografia), Adunanza del 4.12.1872 a Firenze, Roma, Stamperia Reale, 1873.

Atti inchiesta industriale 1873-74b Atti del Comitato dell'inchiesta industriale, vol. III, Deposizioni scritte, categoria 13.2. (incisione, litografia e fotografia), Roma, Tip. Polizzi, 1874, pp. 21-22.

Bertelli / Bollati 1979 Carlo Bertelli / Giulio Bollati, L'immagine fotografica 1845-1945, in Storia d'Italia. Annali, vol. 2, Torino, Einaudi, 1979.

Bollettino Ufficiale 2019 Bollettino Ufficiale della Regione Toscana, n. 51, parte prima, 14 novembre 2019, in <http://raccoltanormativa.consiglio.regione.toscana. it/articolo?urndoc=urn:nir:regione.toscana:legge:2019-11-13;65\&dl_t=text/ xml\&dl_a=y\&dl_id=\&pr=idx,0;artic,0;articparziale,1\&anc=cap4\#top_not4> (07.09.2020).

Caputo Calloud 1992 Annarita Caputo Calloud, Profilo per una storia istituzionale della Società Fotografica Italiana, in "AFT", a. VIII, n. 16, 1992, pp. 17-31.

Cavanna 2020 Pierangelo Cavanna, Il miele e l'argento. Storie di storia della fotografia in Italia, Melfi, Libria, 2020.

Ciuffoletti / Sesti 2003 Zefiro Ciuffoletti / Emanuela Sesti, Il cammino di un'I.D.E.A. Alinari 1920-2002, in Quintavalle/ Maffioli 2003, pp. 239-51.

Ferretti / Conti / Spalletti 2003 Massimo Ferretti / Alessandro Conti / Ettore Spalletti, La documentazione dell'arte, in Settimelli / Zevi 1977, pp. 115-197.

Finito il matrimonio 2012 Finito il matrimonio Alinari- Sole 24ore, in "II Piccolo", 5 settembre 2012, p. 9.

Frosh 2003 Paul Frosh, The Image Factory. Consumer Culture, Photography and the Visual Content Industry, London, Berg, 2003.

Glückler / Panitz 2013 Johannes Glückler / Robert R. Panitz, The Global Stock Image Market. Global Survey 2012. Part I: Players, Products, Business, Heidelberg, GSIM Research Group, 2013.

La serrata 1915 La serrata dello stabilimento fotografico dei Fratelli Alinari, Firenze, Tipografia Barbera, s.d. [ma 1915].

Miraglia 1981 Marina Miraglia, Note per una storia della fotografia italiana (1839-1911), in Storia dell'arte Italiana, vol. 9, tomo II, Grafica e immagine. Illustrazione e fotografia, Torino, Einaudi, 1981, pp. 421-553.

Quintavalle 2003 Arturo Carlo Quintavalle, Gli Alinari, Firenze, Alinari, 2003.

Quintavalle / Maffioli 2003 Arturo Carlo Quintavalle / Monica Maffioli (a cura di), Fratelli Alinari. Fotografi in Firenze. 150 anni che illustrarono il mondo 18522002, Firenze, Alinari, 2003. 
Reberschak 1981 Maurizio Reberschak, Vittorio Cini, in Dizionario Biografico degli Italiani, vol. 25, 1981, ad vocem.

Runge 2020 Evelyn Runge, The Travels of Photographs Within the Global Image Market. How Monopolisation, Interconnectedness, and Differentiation Shape the Economics of Photography, in “Photographies”, vol. 13, 2020, pp. 365-384.

Settimelli / Zevi 1977 Wladimiro Settimelli / Filippo Zevi, Gli Alinari. Fotografi a Firenze 1852-1920, catalogo della mostra (Firenze, Forte Belvedere, 1977), Firenze, Alinari, 1977.

Sisi 2012 Carlo Sisi (a cura di), La commedia dipinta. I concorsi Alinari e il simbolismo in Toscana, catalogo della mostra (Firenze, Museo Marino Marini, 2002-2003), Firenze, Alinari, 2012.

Tomassini 1987 Luigi Tomassini, Gli Alinari e l'editoria fotografica a Firenze fra Ottocento e Novecento, parte II, in "AFT" a. III, n. 6, 1987, p. 62-71.

Tomassini 2013 Luigi Tomassini, L'editoria fotografica e la documentazione del patrimonio artistico italiano. La difficile 'nazionalizzazione' fotografica del Mezzogiorno (1861-1911), in Maria Marcella Rizzo (a cura di), "L'Italia è». Mezzogiorno, Risorgimento e post-Risorgimento, Roma, Viella, 2013, pp. 279306.

Tomassini 2018 Luigi Tomassini, Fotografia e consumi visuali, in Stefano Cavazza / Emanuela Scarpellini (a cura di), Storia d'Italia. Annali, vol. 27, Torino, Einaudi, 2018, pp. 595-620.

Zannier 1982 Italo Zannier, Storia e tecnica della fotografia, Bari-Roma, Laterza, 1982.

\#Archivio Cini Archivio della Fondazione Giorgio Cini, b. 3.

\#ASAI 1929 Archivio Storico Alinari IDEA, Firenze, Libro dei verbali del CdA, Seduta del 10 aprile 1929.

\#ASAI 1957 Archivio Storico Alinari IDEA, Firenze, Libro dei verbali del CdA, Seduta del 4 maggio 1957.

\#ASAI 1958 Archivio Storico Alinari IDEA, Firenze, Verbale dell'assemblea del 10 dicembre 1958.

\#ASAI 1960 Archivio Storico Alinari IDEA, Firenze, Bilancio 1960. 